\title{
SirT1 and STAT3 protect retinal pigmented epithelium cells against oxidative stress
}

\author{
LANGEN LI $^{1}$, WEI WEI ${ }^{2}$, YUFENG ZHANG ${ }^{2}$, GERILE TU $^{2}$, YANMEI ZHANG $^{3}$, JIA YANG $^{4}$ and YIQIAO XING ${ }^{1}$ \\ ${ }^{1}$ Department of Ophthalmology, Renmin Hospital of Wuhan University, Wuhan, Hubei 430060; \\ Departments of ${ }^{2}$ Ophthalmology and ${ }^{3}$ Neurology, Inner Mongolia People's Hospital, Hohhot, Inner Mongolia 010017; \\ ${ }^{4}$ Department of Neurology, The Affiliated Hospital of Inner Mongolia Medical University, \\ Hohhot, Inner Mongolia 010017, P.R. China
}

Received April 16, 2014; Accepted February 19, 2015

DOI: $10.3892 / \mathrm{mmr} .2015 .3570$

\begin{abstract}
It has been previously demonstrated that there are interactions between sirtuin 1 (SirT1) and signal transducer and activator of transcription 3 (STAT3), which have versatile roles in various microenvironments. However, whether or not there is crosstalk between these two molecules during oxidative stress, and what mechanism of crosstalk occurs in retinal pigmented epithelium cells (RPEs), the protection of which may delay the process of age-related macular degeneration (AMD), has required further elucidation. The present study aimed to investigate the interactions between SirT1 and STAT3 in RPEs, following exposure to oxidative stress. The rates of proliferation and apoptosis, levels of intracellular reactive oxygen species and cell senescence of RPEs, induced by oxidants $\left[\mathrm{H}_{2} \mathrm{O}_{2}\right.$ and oxidized low density lipoprotein (oxLDL)], were evaluated. The results revealed a downregulation of SirT1 expression, and an upregulation of STAT3 expression during oxidative stress. Further investigation indicated that SirT1 protected RPEs from oxidative stress-induced damage. Furthermore, gain- and loss-of-function experiments indicated that SirT1 had negative effects on the regulation of STAT3 expression in RPEs during oxidative stress. Notably, STAT3 directly protected the cells from oxidative stress, rather than depending on SirT1. Additionally, the protective effects of STAT3 had no association with the modulation of cell senescence during oxidative stress. In conclusion, SirT1 had negative effects on the regulation of STAT3 expression during oxidative stress. However, SirT1 and STAT3 demonstrated protective roles against oxidative stress in RPEs. These results therefore suggested that there was an equilibrium mechanism between SirT1 and STAT3 against oxidative stress, meaning that an
\end{abstract}

Correspondence to: Dr Yiqiao Xing, Department of Ophthalmology, Renmin Hospital of Wuhan University, 99 Zhangzhidong Road, Wuhan, Hubei 430060, P.R. China

E-mail: yqxingwh@sohu.com

Key words: signal transducer and activator of transcription 3, sirtuin 1, oxidative stress, age-related macular degeneration, ophthalmology equilibrium mechanism is required to be considered when combined application of STAT3 and SirT1 were performed to treat AMD.

\section{Introduction}

Age-related macular degeneration (AMD) is the most prevalent cause of central vision loss amongst elderly individuals $(1,2)$. It has been reported that smoking cigarettes is the greatest environmental risk factor for the development of AMD (3). The mechanism underlying the effect of smoking on the incidence and severity of AMD remains to be elucidated. However, it is clear that cigarettes contain a large number of oxidants (4). The Age-Related Eye Disease Study Group demonstrated that antioxidants are able to reduce the risk of individuals proceeding to advanced AMD (5). Furthermore, proteomic analysis identified numerous proteins in the drusen, which developed as a result of oxidative damage (6). It was therefore hypothesized that oxidative stress may be involved in AMD pathogenesis, and that oxidative injury contributes to the pathogenesis of AMD (1,7). According to postmortem analyses, patients with AMD exhibit extensive free radical expression and damage to the proteins, lipids, DNA and mitochondria of retinal pigment epithelial cells (RPEs) (8), further supporting this hypothesis. RPEs are a single layer of epithelial cells, which are located between the light-sensing photoreceptor cells and the choriocapillaris. Damage to the RPEs is known to be an early event in AMD (9). Oxidative stress is implicated in the development of age-associated RPE cell degeneration, dysfunction and loss $(10,11)$. Therefore the elucidation of effective methods for protecting RPEs from oxidative stress in order to delay the onset and/or progression of AMD is required (12).

Cells have certain protective strategies, which minimize the effects of oxidative damage. The most well-documented of these defense mechanisms is the production of endogenous antioxidants, including superoxide dismutases (SODs), carotenoids and vitamins (7). Additionally, specific cytoprotective proteins participate in combating oxidative stress, for example sirtuin 1 (SirT1) and signal transducer and activator of transcription 3 (STAT3). SirT1, a class III protein deacetylase, is a longevity factor and an aldehyde dehydrogenase-dependent protein deacetylase, the activity of which has regulatory 
effects on cellular stressors, including genotoxic, oxidative and proteotoxic stress $(13,14)$. It has been reported that moderate overexpression of SirT1 protects the mouse cardiac muscle against oxidative stress and attenuates the development of age-associated cardiomyopathy involving hypertrophy (15). SirT1 is also able to inhibit certain transcription factors, which regulate cellular redox balance. For example, inhibition of the transactivation capacity of nuclear factor-like 2 (Nrf2) in HepG2 cells (16). However the detailed mechanisms underlying the function of SirT1 against oxidative stress, particularly in RPEs, remain to be elucidated. STAT3, one of the seven STATs, is regulated by a signaling regulator, comprising interleukin (IL)-6 and IL-10 family members, and has multiple functions in mediating cell survival, apoptosis, migration and differentiation (17). Regarding oxidative stress, previous studies have demonstrated the crosstalk between STAT3 and oxidative metabolism and its involvement in cancer and heart disease $(18,19)$. Based on the known roles of SirT1 and STAT3 in oxidative stress, it was hypothesized that there is crosstalk between the two molecules during oxidative stress. Interactions between SirT1 and STAT3, which have versatile roles in various microenvironments, had previously been identified $(20,21)$. However, whether or not there was crosstalk between the two molecules during oxidative stress in RPEs and what the mechanism of crosstalk was, remained to be elucidated.

In the present study, the proliferation, apoptosis, intracellular ROS and senescence of RPEs induced by oxidants, as well as the expression of SirT1 and STAT3 during oxidative stress were evaluated. Subsequently, the effects of SirT1 on proliferation, apoptosis, intracellular ROS and senescence of RPEs during oxidative stress was examined. Finally, the SirT1/STAT3 interaction in RPEs upon exposure to oxidative stress was investigated.

\section{Materials and methods}

Cell culture. The ARPE-19 human RPE cell line was purchased from the American Type Culture Collection (Manassas, VA, USA) and grown in Dulbecco's modified Eagle's medium (DMEM) containing 10\% heat-inactivated fetal bovine serum (FBS; Gibco, Invitrogen Life Technologies, Carlsbad, CA, USA), $100 \mu \mathrm{g} / \mathrm{ml}$ streptomycin and $100 \mathrm{U} / \mathrm{ml}$ penicillin (Harbin Pharmaceutical Group Co., Ltd., Harbin, China) in $5 \% \mathrm{CO}_{2}$ at $37^{\circ} \mathrm{C}$. The cells were passaged approximately every 3 days and subcultured at $\sim 90 \%$ confluence.

Cell proliferation and apoptosis assay. ARPE-19 cells were grown to $80 \%$ confluence, at 2000 cells/well in 96-well plates. Cells were subsequently treated with oxidized low density lipoprotein (oxLDL; $200 \mu \mathrm{g} / \mathrm{ml}$; Kalen Biomedical, Montgonery Village, MD, USA) or $\mathrm{H}_{2} \mathrm{O}_{2}(50 \mu \mathrm{M})$ for $24 \mathrm{~h}$. Proliferation and apoptosis were assessed using a Cell Titer $96^{\circledR}$ AQueous One Solution reagent (Promega Corporation, Madison, WI, USA) and an Annexin V-fluorescein isothiocyanate apoptosis detection kit (Sigma-Aldrich, St. Louis, MO, USA) according to the manufacturer's instructions. Briefly, $1-5 \times 10^{5}$ cells were resuspended in $0.5 \mathrm{ml}$ binding buffer and incubated with annexin $\mathrm{V}$-fluorescein isothiocyanate and propidium iodide for $10 \mathrm{~min}$ in the dark at room temperature. A FACScan flow cytom- eter (BD Biosciences) equipped with a FITC signal detector FL1 (excitation $488 \mathrm{~nm}$ ) and a phycoerythrin emission signal detector FL3 (excitation $585 \mathrm{~nm}$ ) was used to analyze cellular apoptosis. The results were calculated using the CellQuestTM Pro software (BD Biosciences) and expressed as the percentage of apoptotic cells from the total cells.

Analysis of intracellular reactive oxygen species (ROS) levels. Intracellular ROS levels were measured using an intracellular ROS assay kit (Cell Biolabs, San Diego, CA, USA). Briefly, ARPE-19 cells were incubated with 2',7'-dichlorodihydro fluorescin diacetate (DCFH-DA) in the culture medium for $30 \mathrm{~min}$ at $37^{\circ} \mathrm{C}$. Subsequently, the pre-loaded cells were treated with oxLDL $(200 \mu \mathrm{g} / \mathrm{ml})$ or $\mathrm{H}_{2} \mathrm{O}_{2}(50 \mu \mathrm{M})$ for $24 \mathrm{~h}$ or as described in the figure legends. DCFH fluorescence of the cell lysate was observed by using fluorescence microscope (OLYMPUS, Japan). Imags were captured using a Digital SLR camera (Canon, Beijing, China). The fluorescence intensity of each reaction mixture was determined and quantified using Image $\mathbf{J}$ software v1.4 (National Institutes of Health, Bethesda, MA, USA).

Cell senescence assay. Cell senescence was measured using a cellular senescence assay kit associated $\beta$-gal (SA- $\beta$-gal) assay (Cell Biolabs, San Diego, CA, USA), assay according to the manufacturer's instructions. Briefly, ARPE-19 cells were cultured to 2000 cells/well in 96-well plates, and then exposed to oxLDL $(200 \mu \mathrm{g} / \mathrm{ml})$ or $\mathrm{H}_{2} \mathrm{O}_{2}(50 \mu \mathrm{M})$ for $24 \mathrm{~h}$ in a $37^{\circ} \mathrm{C}$ incubator. Following washing and collection, cells were treated with freshly prepared fluorometric substrate for $2 \mathrm{~h}$ at $37^{\circ} \mathrm{C}$ in the dark. The fluorescence intensity of each reaction mixture was determined and quantified using Image $\mathbf{J}$ software (NIH). The average fluorescence intensity was analyzed from five fields for each treatment.

Cell treatments. Cells were incubated with $\mathrm{H}_{2} \mathrm{O}_{2}(50 \mu \mathrm{M})$ for $24 \mathrm{~h}$. Subsequently, $\mathrm{H}_{2} \mathrm{O}_{2}$ was detoxified by the addition of catalase (100,000 $\mu \mathrm{l}$; Worthington Biochemical Corp., Lakewood, NJ, USA) at the end of the incubation (22). oxLDL $(200 \mu \mathrm{g} / \mathrm{ml})$ was added to other samples to induce pathological stress in ARPE-19 cells following $24 \mathrm{~h}$ treatment (23). To further evaluate the effects of SirT1, resveratrol (RSV; $10 \mathrm{mM}$ ) and nicotinamide (NA; $5 \mathrm{mM}$ ) (Sigma-Aldrich) were applied to incubated cells prior to oxidant treatments (24). The pRC/CMV-STAT3 and pcRC/CMV expression vectors (STAT3OV and C-STAT3OV) were acquired from Promega Corporation and used to evaluate the effects of STAT3 overexpression. SirT1 and STAT3 knockdown were performed using small interfering (si)RNA for SirT1 (Santa Cruz Biotechnology Inc., Dallas, TX, USA) and short hairpin (sh)RNA STAT3/Puro (STAT3KO; Sigma-Aldrich) lentiviral vectors, respectively. shRNAi Mission Non-Target shRNA Control/Puro (Sigma-Aldrich) was used as a negative control of gene knockdown (C-SirT1KO and C-STAT3KO). Cell transfection was performed using Lipofectamine ${ }^{\circledR} 2000$ reagent (Invitrogen Life Technologies) according to the manufacturer's instructions.

RNA extraction and reverse transcription-quantitative polymerase chain reaction $(R T-q P C R)$. Total RNA was extracted using RNeasy kits (Qiagen, Valencia, CA, USA) and first-strand complementary DNA was synthesized using 
A

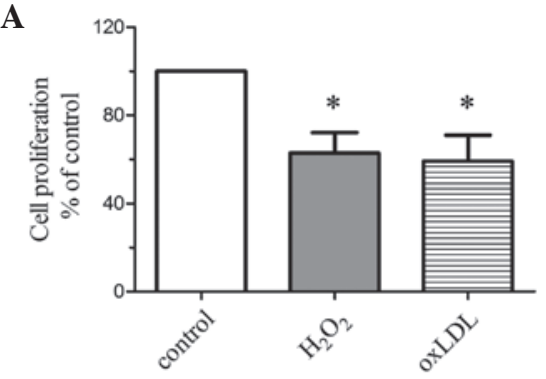

C

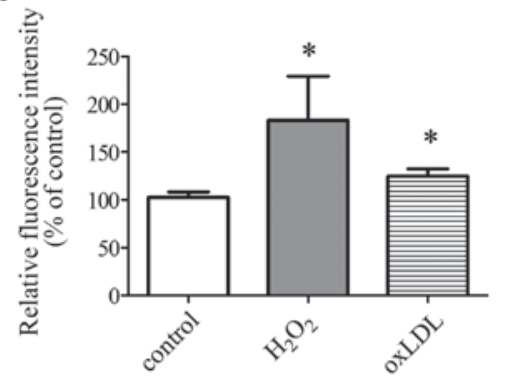

E

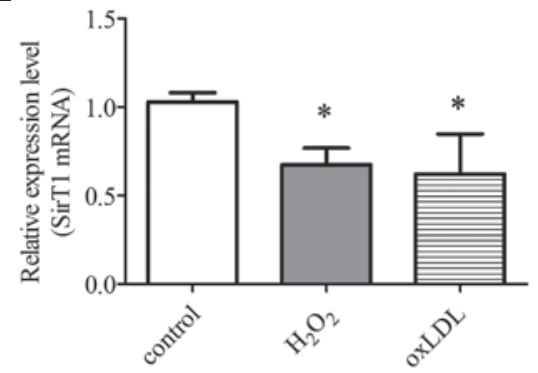

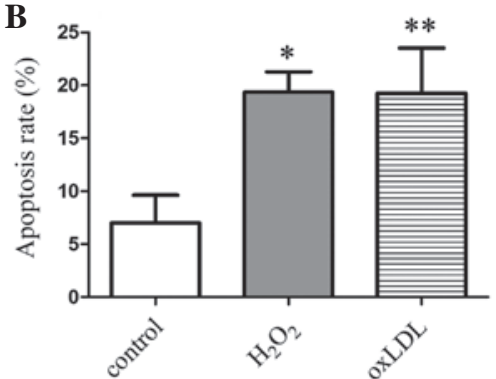

D

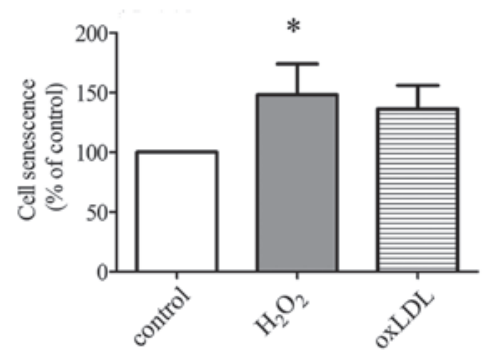

F

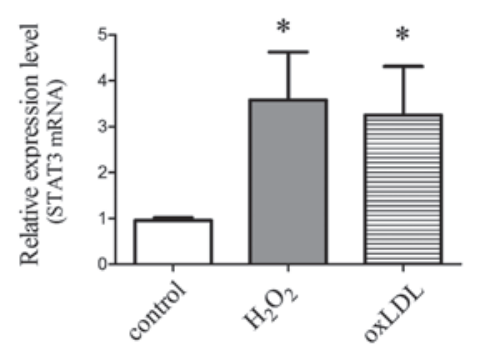

Figure 1. Effects of oxidants $\mathrm{H}_{2} \mathrm{O}_{2}$ and oxLDL on RPEs. ARPE-19 cells were grown at $80 \%$ confluence and exposed to $\mathrm{H}_{2} \mathrm{O}_{2}(50 \mu \mathrm{M})$ or oxLDL (200 $\left.\mu \mathrm{g} / \mathrm{ml}\right)$ for $24 \mathrm{~h}$. Subsequently, (A) proliferation, (B) apoptosis, (C) intracellular ROS and (D) senescence of RPEs were assessed. The expression of SirT1 and STAT3 mRNA was evaluated by reverse transcription-quantitative polymerase chain reaction. Ct values were normalized using the $2^{-\Delta \Delta C t}$ method as relative quantification as shown in (E) SirT1 and (F) STAT3. Values are expressed as the mean \pm standard deviation of three independent experiments. Analysis of variance was performed to analyze the differences statistically. ${ }^{*} \mathrm{P}<0.05,{ }^{* *} \mathrm{P}<0.01$ vs. control. OxLDL, oxidized low density lipoprotein; RPEs, retinal pigmented epithelium cells; ROS, reactive oxygen species; mRNA, messenger RNA; SirT1, sirtuin 1; STAT3, signal transducer and activator of transcription 3.

SuperScript III (Invitrogen Life Technologies). RT-qPCR was performed on an ABI 7500 (Applied Biosystems Life Technologies, Foster City, CA, USA) with a SYBR Premix Ex Taq ${ }^{\mathrm{TM}}$ kit (Takara Bio, Inc., Otsu, Japan). GAPDH was used as the internal control. Data were normalized using the $2^{-\Delta \Delta \mathrm{Ct}}$ method for relative quantification. The primers were as follows: Forward: GGGTGGAGAAGGACATCAGCGGTAA and reverse: GCCGACAATACTTTCCGAATCC for STAT3, forward: TGTGGTAGAGCTTGCATTGATCTT and reverse: GGCCTGTTGCTCTCCTCAT for SirT1 and forward: GGAGTCAACGGATTTGGTC and reverse: GGAATCATTGGAACATGTAAAC for GAPDH. The reactions began at $95^{\circ} \mathrm{C}$ for $5 \mathrm{~min}$, followed by 40 cycles of $95^{\circ} \mathrm{C}$ for $15 \mathrm{sec}$ and $60^{\circ} \mathrm{C}$ for $30 \mathrm{sec}$, and extension for $1 \mathrm{~min}$ at $72^{\circ} \mathrm{C}$.

Western blot analysis. Cell lysates were acquired from the collected cells cell lysis reagent (Promega, Madison, WI, USA), containing Complete Mini protease inhibitor cocktail (Roche). The protein concentration was determined by using Bradford Reagent (Bio-Rad) and the protein samples were subjected to $10 \%$ SDS-PAGE. The proteins were subsequently transferred onto nitrocellulose membranes and the membranes were blocked with $50 \mathrm{mM}$ Tris- $\mathrm{HCl}(\mathrm{pH} 7.5)$,
$150 \mathrm{mM} \mathrm{NaCl}$ and $0.05 \%$ Tween-20 (TBST), containing 5\% non-fat milk or 5\% bovine serum albumin (Sigma-Aldrich) for $30 \mathrm{~min}$. The membranes were subsequently incubated with rabbit monoclonal primary antibodies against STAT3 (1:500; Minneapolis, MI, USA), phosphospecific STAT3 (Tyr705) (1:500; Cell Signaling Technology, Inc., Danvers, MA, USA), SirT1 (1:500; Sigma-Aldrich) and mouse $\beta$-actin (1:1,000; Sigma-Aldrich) overnight at $4^{\circ} \mathrm{C}$. Subsequently, goat anti-mouse immunoglobulin $\mathrm{G}$ (IgG) or goat anti-rabbit IgG (Pierce Biotechnology, Inc., Thermo Fisher Scientific, Rockford, IL, USA) were utilized to visualize the results, which were detected using enhanced chemiluminescence detection systems (Super Signal West Femto; Pierce Biotechnology, Inc.).

Statistical analysis. Values are presented as the mean \pm standard deviation. Student's t-test was used to analyze differences between two groups. The experimental results were analyzed with one-way analysis of variance to compare the differences between three or more groups. $\mathrm{P}<0.05$ was considered to indicate a statistically significant difference. Statistical analyses were conducted using SPSS 17.0 software (SPSS, Chicago, IL, USA). All experiments were performed in triplicate. 

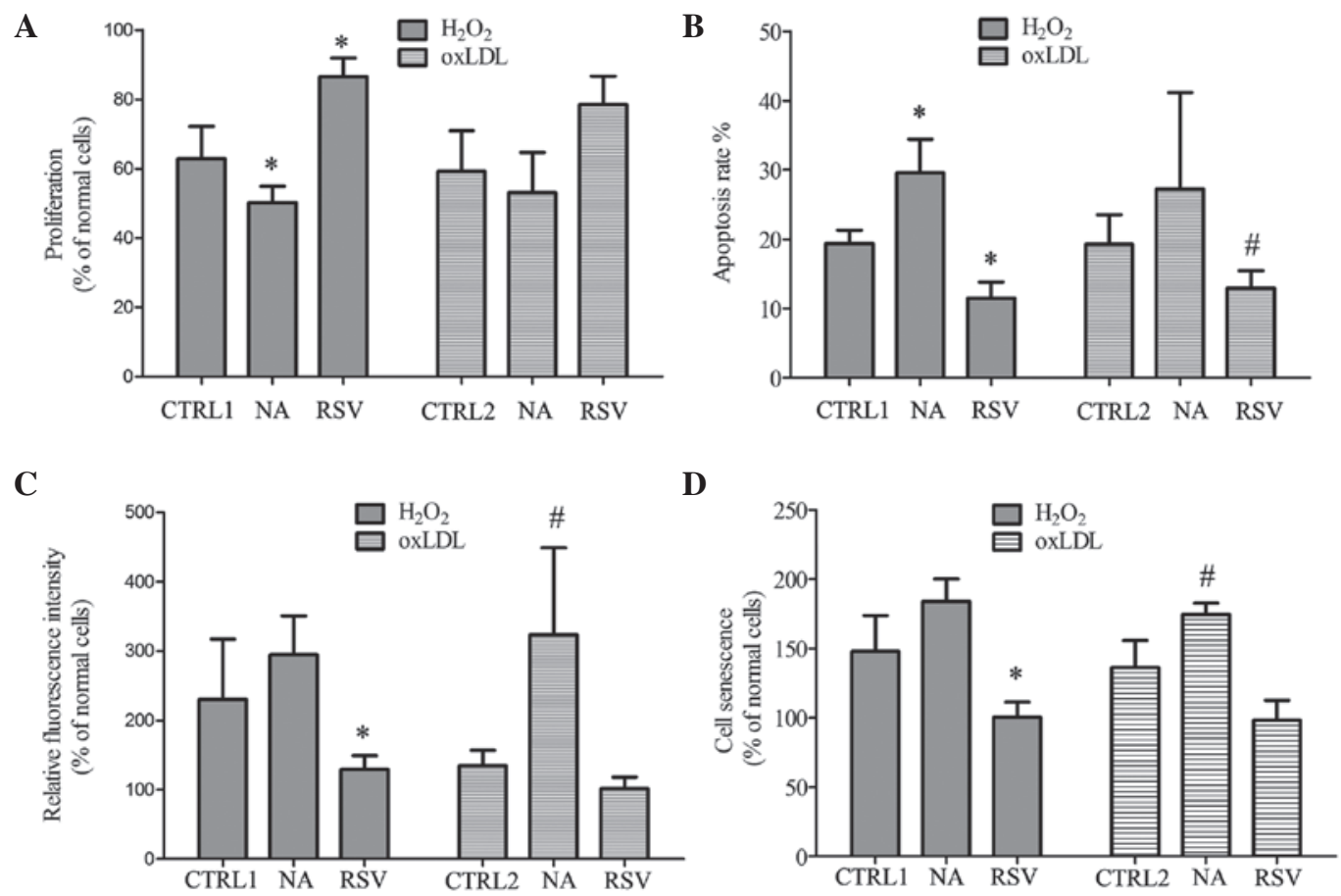

Figure 2. SirT1 protect RPEs from the damage induced by oxidative stress. ARPE-19 cells were treated with $\mathrm{H}_{2} \mathrm{O}_{2}(50 \mu \mathrm{M})$ or oxLDL (200 $\left.\mu \mathrm{g} / \mathrm{ml}\right)$ for $24 \mathrm{~h}$, following incubation with $10 \mathrm{mM}$ RSV or $5 \mathrm{mM} \mathrm{NA}$ for $4 \mathrm{~h}$. (A) Proliferation was evaluated using Cell Titer 96 AQueous One Solution reagent. The data was normalized according to the proliferation rate of untreated normal cells. (B) Apoptotic rate of cells was detected by a TUNEL assay. (C) Intracellular ROS were measured by 2',7'-dichlorodihydro fluorescin diacetate. The data was normalized according to the intracellular ROS of untreated normal cells. (D) Cell senescence was evaluated by an SA- $\beta$-gal assay. Data in each panel are from three independent experiments. " $\mathrm{P}<0.05$ vs. CTRL1, "P $<0.05$ vs. CTRL2. CTRL1, cells treated with $\mathrm{H}_{2} \mathrm{O}_{2}$; CTRL2, cells treated with oxLDL; RSV, resveratrol; NA, nicotinamide; SirT1, sirtuin 1; RPEs, retinal pigmented epithelium cells; oxLDL, oxidized low density lipoprotein; ROS, reactive oxygen species.

\section{Results}

Oxidative stress induces RPE damage and dysregulation of SirT1 and STAT3. In order to mimic the oxidative damage that occurs in AMD, $\mathrm{H}_{2} \mathrm{O}_{2}$ and oxLDL have been previously employed as experimental oxidants, with which to induce pathological stresses in RPEs $(25,26)$. Following treatment with $\mathrm{H}_{2} \mathrm{O}_{2}(50 \mu \mathrm{M})$ or oxLDL $(200 \mu \mathrm{g} / \mathrm{ml})$ for $24 \mathrm{~h}$, the proliferation, apoptosis, intracellular ROS and senescence of ARPE-19 cells were analyzed. It was revealed that $\mathrm{H}_{2} \mathrm{O}_{2}$ and oxLDL significantly elevated cellular proliferation (Fig. 1A; $\mathrm{P}=0.020$ and $\mathrm{P}=0.026$, respectively) and significantly enhanced the apoptotic rate (Fig. 1B; $\mathrm{P}=0.013$ and $\mathrm{P}=0.003$, respectively), compared with that of the control group. Due to the association of an accumulation of intracellular ROS with AMD (27), the intracellular ROS levels were also evaluated. $\mathrm{H}_{2} \mathrm{O}_{2}$ and oxLDL significantly increased the intracellular ROS levels in RPEs ( $\mathrm{P}=0.040$ and $\mathrm{P}=0.016$, respectively), compared with those of the control group (Fig. 1C). Cellular senescence has a critical function in contributing to AMD, and the biological ageing process is associated with an increase in senescence (25). The cellular senescence of RPEs induced by $\mathrm{H}_{2} \mathrm{O}_{2}$ or oxLDL was evaluated using an SA- $\beta$-gal assay. As indicated in Fig. 1D, oxLDL and $\mathrm{H}_{2} \mathrm{O}_{2}$ promoted cell senescence, compared with the control group, with a significant difference following treatment with $\mathrm{H}_{2} \mathrm{O}_{2}(\mathrm{P}=0.045)$.

To analyze the effects of SirT1 and STAT3 on oxidative stress, the expression of SirT1 and STAT3 in RPEs was assessed. Notably, the expression of SirT1 mRNA was found to be downregulated in RPEs treated with $\mathrm{H}_{2} \mathrm{O}_{2}$ and oxLDL, when compared with that in the control group $(\mathrm{P}=0.005$ and $\mathrm{P}=0.039$, respectively). Conversely, the expression of STAT3 mRNA was demonstrated to be upregulated $(\mathrm{P}=0.012$ and $\mathrm{P}=0.020$, respectively), as shown in Fig. 1E and F. These results demonstrated that oxidative stress induced RPE damage and resulted in the dysregulation of SirT1 and STAT3 mRNA expression.

SirT1 influences the damage of RPEs induced by oxidative stress. In order to evaluate the effects of SirT1 in oxidative stress, RPEs were pretreated with the SirT1 inhibitor, NA, or the SirT1 activator, RSV, prior to treatment with $\mathrm{H}_{2} \mathrm{O}_{2}(50 \mu \mathrm{M})$ or oxLDL $(200 \mu \mathrm{g} / \mathrm{ml})$ for $24 \mathrm{~h}$. The proliferation (Fig. 2A), apoptosis (Fig. 2B), intracellular ROS (Fig. 2C) and senescence (Fig. 2D) of RPEs were subsequently evaluated. NA enhanced the effects of $\mathrm{H}_{2} \mathrm{O}_{2}$, markedly decreasing the proliferation rate $(\mathrm{P}=0.049)$, stimulating apoptosis $(\mathrm{P}=0.028)$, as well as increasing intracellular ROS and senescence in RPEs. Conversely, RSV was able to resist the effects of $\mathrm{H}_{2} \mathrm{O}_{2}$. To examine whether SirT1 had analagous effects on RPEs treated with oxLDL, identical experiments were performed following oxLDL treatment. Furthermore, the SirT1 activator, RSV, exerted opposite effects on the regulation of RPEs, when compared with the effects of NA, as indicated in Fig. 2.

SirT1 negatively influences the expression of STAT3 during oxidative stress. In the present study, an overexpression of STAT3, which was involved in STAT3 activation during oxidative stress in RPEs, was identified. In order to better understand 
A

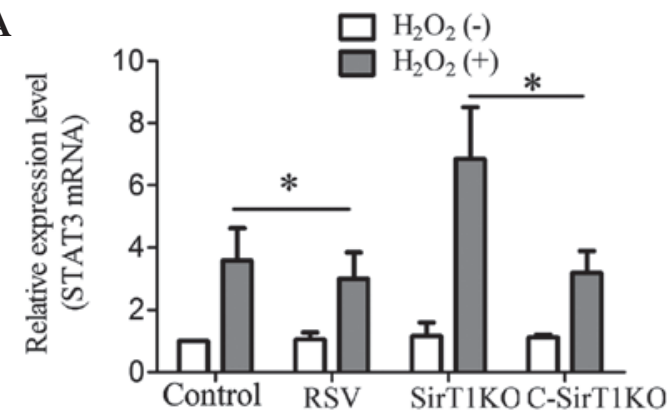

C

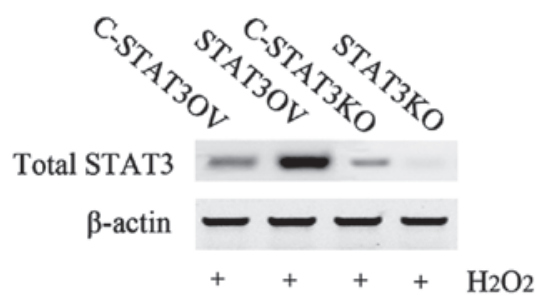

B

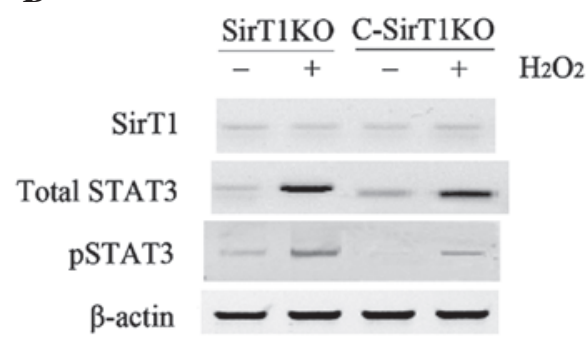

D

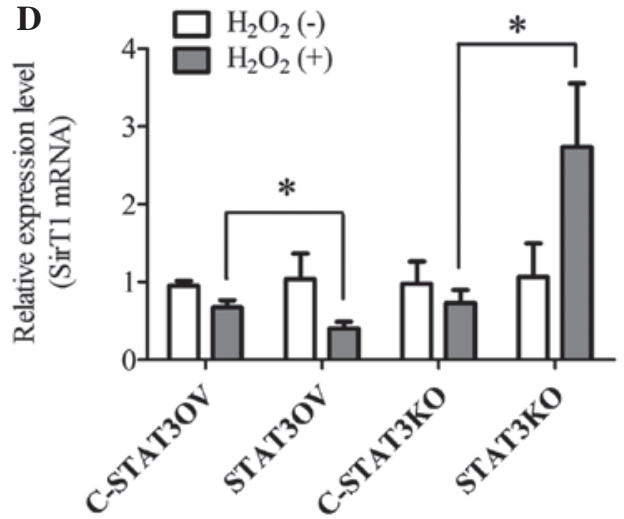

Figure 3. SirT1 counteracts the expression of STAT3 in RPEs during oxidative stress. ARPE-19 cells were pretreated with 10 mM RSV (RSV group), small interfering RNA for SirT1KO group or C-SirT1KO group. Subsequently, pretreated cells were exposed to $\mathrm{H}_{2} \mathrm{O}_{2}$ for 24 h. (A) Relative expression of STAT3 mRNA was detected by reverse transcription-quantitative polymerase chain reaction. (B) Western blot analysis demonstrated the efficiency of SirT1 knockdown, as well as the expression levels of STAT3 protein and pSTAT3 in the SirT1KO and C-SirT1KO groups. (C) Silencing of STAT3 was confirmed by western blot analysis. (D) Expression levels of SirT1 were compared between the STAT3OV and C-STAT3OV groups, and between the STAT3KO and C-STAT3KO groups. Values are expressed as the mean \pm standard deviation of three independent experiments, ${ }^{*} \mathrm{P}<0.05$. SirT1, sirtuin 1 ; STAT3, signal transducer and activator of transcription 3 ; RSV, resveratrol; sirT1KO, SirT1 knockdown; C-SirT1KO, non-target short hairpin RNA; STAT3OV, STAT3 overexpression; C-STAT3OV, control expression vector; mRNA, messenger RNA; pSTAT3, phosphorylated STAT3.

the protective roles of SirT1 in RPEs during oxidative stress, the association between STAT3 activation and SirT1 required elucidation. Differences in the expression of STAT3 following SirT1 knockdown, or overexpression in RPEs during oxidative stress were evaluated. As shown in Fig. 3A, the SirT1 activator, RSV, significantly decreased the expression of STAT3 $(\mathrm{P}=0.048)$, while a significant increase in STAT3 expression was detected in RPEs following SirT1 knockdown $(\mathrm{P}=0.025)$, compared with that in the control, during $\mathrm{H}_{2} \mathrm{O}_{2}$ treatment. Furthermore, SirT1 knockdown was also found to promote the expression of STAT3 protein and phosphorylated STAT3 protein during oxidative stress (Fig. 3B), whereas RSV and SirT1 knockdown did not influence the expression of STAT3 in the absence of oxidative stress (Fig. 3A and B). The results presented in Fig. 3B demonstrate the efficiency of SirT1 knockdown. To further investigate the association between STAT3 and SirT1, the expression of SirT1 following STAT3 knockdown (STAT3KO group) or overexpression (STAT3OV group) was evaluated in RPEs during oxidative stress. The expression of STAT3 protein was determined in each group by western blot analysis, as shown in Fig. 3C. Notably, overexpression of STAT3 induced a decrease in the expression of SirT1, when compared with that in the C-STAT3OV group $(\mathrm{P}=0.022)$, during oxidative stress. The expression of SirT1 was significantly enhanced in the STAT3KO group (Fig. 3D; P=0.015). These results indicated that STAT3 exerts negative effects on the expression of SirT1 in RPEs during oxidative stress.
STAT3 protects ARPE-19 cells from oxidative stress, independent of the regulation of cell senescence. STAT3 activation has been associated with the protection of retinal cells during injury, including that induced by oxidative stress (28). Therefore, in order to confirm the association between the effects of STAT3 in the protection of cells and the protective roles of SirT1 during oxidative stress, the effects of STAT3 on oxidative stress following pretreatment with the SirT1 inhibitor, NA, were assessed. As shown in Fig. 4, STAT3 increased cell proliferation (Fig 4A), decreased apoptosis (Fig. 4B) and increased intracellular ROS (Fig. 4C) in the presence of NA. However, STAT3 did not influence cell senescence (Fig. 4D). These results revealed that STAT3 directly protected the cells from oxidative stress rather than depending on SirT1 activity. They also suggested that the protective effects of STAT3 were not associated with the modulation of cellular senescence during oxidative stress. Notably, these results suggested that there is an equilibrium mechanism between the functions of SirT1 and STAT3 against oxidative stress, due to their specific interactions and effects in RPEs.

\section{Discussion}

Oxidative stress is considered an important contributing factor to the development of $\mathrm{AMD}$, and has been implicated in the pathophysiology of AMD $(1,7)$. The protection of RPEs against oxidative damage presents a potential therapeutic strategy 
A

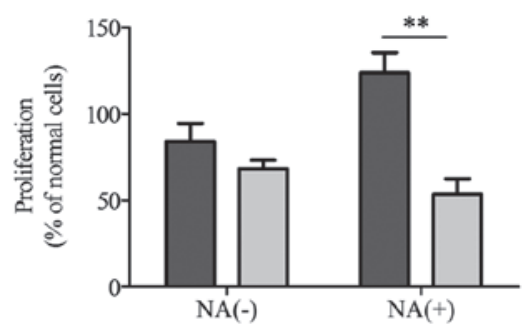

C

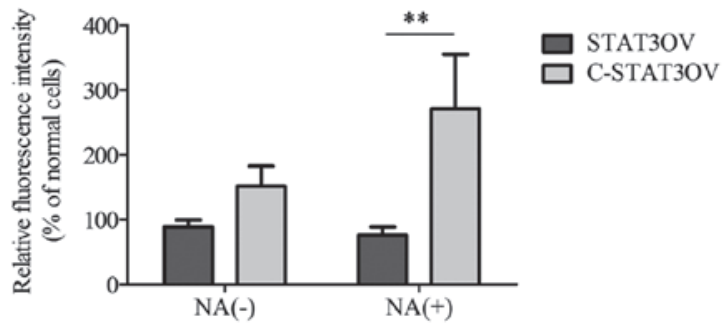

B

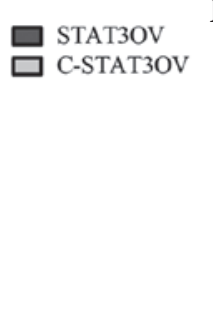

D
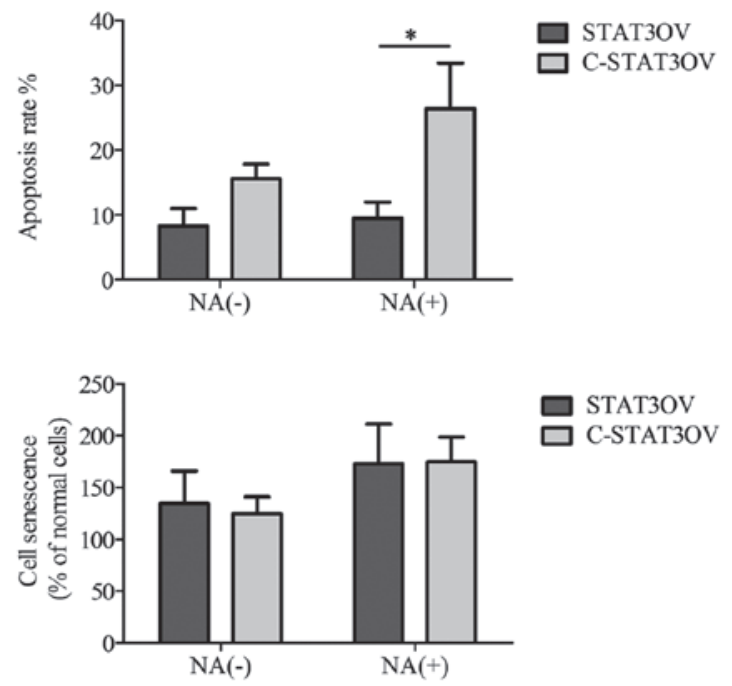

Figure 4. STAT3 directly protects ARPE-19 cells from oxidative stress. ARPE-19 cells were transfected with pRC/CMV-STAT3 (STAT3OV group) or pcRC/CMV (C-STAT3OV group). Cells were subsequently treated with $\mathrm{H}_{2} \mathrm{O}_{2}$ for 24 h following incubated with 5 mM SirT1 inhibitor NA for 4 h. (A) Proliferation, (B) apoptosis, (C) intracellular reactive oxygen species and (D) senescence of retinal pigmented epithelium cells were assessed and compared amongst these groups. Data in each panel are from three independent experiments. ${ }^{*} \mathrm{P}<0.05,{ }^{* *} \mathrm{P}<0.01$. STAT3, signal transducer and activator of transcription 3 ; SirT1, sirtuin 1; NA, nicotinamide.

for AMD (12). $\mathrm{H}_{2} \mathrm{O}_{2}$ has been extensively used to mimic oxidative stress in vitro. $\mathrm{H}_{2} \mathrm{O}_{2}$ and oxLDL were therefore added to cultured RPEs in order to mimic oxidation-induced AMD injury $(26,27,29)$. However, the detailed mechanisms underlying the injuries induced by $\mathrm{H}_{2} \mathrm{O}_{2}$ and oxLDL, and a comparison between the effects of $\mathrm{H}_{2} \mathrm{O}_{2}$ and oxLDL, which were used as oxidants to mimic oxidative stress in RPEs, remained to be elucidated. In the present study, a model to test oxidative stress susceptibility and antioxidant efficiency in RPEs was constructed. It was found that two oxidants $\left(\mathrm{H}_{2} \mathrm{O}_{2}\right.$ and oxLDL) induced damage to RPEs, including attenuation of proliferation, increased apoptosis, increased intracellular ROS and promotion of cell senescence.

SirT1 exerts multiple, pleiotropic effects in oxidative metabolism (30-32). In addition to post-translational modifications induced by oxidative stress (33), SirT1 has been demonstrated to protect cells against oxidative stress via the upregulation of antioxidant genes and the modulation of Nrf2 (16). SirT1 expression and activity levels are decreased during lung inflammation caused by oxidative stress, and in human endothelial cells and macrophages, following exposure to oxidants $(34,35)$. However, whether SirT1 levels remain decreased in RPEs under oxidative stress required experimental investigation. In the present study, it was shown that $\mathrm{H}_{2} \mathrm{O}_{2}$ and oxLDL decreased SirT1 levels in RPEs. STAT3, which was activated at a basal level in the RPEs, was upregulated and activated following oxidative stress-induced RPE injury $(36,37)$. The results of the present study were in accordance with those of previous studies $(36,37)$. Dysregulation of SirT1 and STAT3 implied that the two molecules may have important roles in the modulation of oxidative stress in RPEs.

It has previously been reported that moderate overexpression of SirT1 may have protective roles in the heart, following oxidative stress-induced injury (15). In addition, SirT1 was previously reported to exert significant protective effects against ocular diseases in animal models, including cataracts, retinal degeneration, optic neuritis and uveitis (38). Regarding the protective roles of SirT1 in RPEs, it has been demonstrated that SirTl may be a significant regulator in the protection of RPEs against ultraviolet B-induced injury, and may abrogate amyloid- $\beta$-induced inflammation $(39,40)$. In the present study, SirT1 was demonstrated to significantly attenuate the effects induced by oxidants. These results indicated that SirT1 may provide protection against oxidative stress in animal models and RPEs.

Bernier et al (22) reported negative regulation of STAT3 protein-mediated cellular respiration by SirT1 in murine embryonic fibroblast cells (21), and a previous study provided further support for these results (41). As previously demonstrated, pSTAT3 stimulated expression of the STAT3 gene [unphosphorylated STAT3 (U-STAT3)], which exerted persistent activity (42). The STAT3 gene is driven by pSTAT3 (phosphorylated STAT3) (1). pSTAT3 lead to dramatically increased U-STAT3 (Unphosphorylated STAT3) which persists for many days. Nevertheless pSTAT3 is sustained for a short period of time $(2,3)$. In the present study, the effects of pSTAT3 on SirT1 were not investigated; instead, the expression levels of total STAT3 mRNA, which reflected the expression of U-STAT3 and pSTAT3, were analyzed. The level of total STAT3 may be in response to the degree of STAT3 activation. The use of gain- and loss-of-function experiments revealed that SirT1 exerted negative effects on the regulation of STAT3 expression during oxidative stress in RPEs (Fig. 3).

STAT3 is an essential mediator of Toll-like receptor 3-regulated protection of RPEs against oxidative stress (20). STAT3 was shown to be a directly protective regulator in the present study, and the protective effects of SirT1 against oxidative stress in RPEs were also demonstrated. It is therefore hypothesized that SirT1 and STAT3 may exert synergistic effects against oxidative stress. SirT1 attenuated STAT3 activation according to the results of a previous study (43), and those of the present study. The present study examined how SirT1 regu- 
lated STAT3, in order to protect RPEs against oxidative stress. The results of the present study revealed that the protective effects of STAT3 were increased in the presence of the SirT1 inhibitor NA; although, no alterations in cellular senescence were detected. These results suggested that SirT1 had crucial roles in protection against oxidative stress in the presence of SirT1 activator, while STAT3 was an essential protective regulator when the effect of SirT1 was inhibited. There may therefore be an equilibratory mechanism between SirT1 and STAT3. Alcendor et al (15) demonstrated that moderate (7.5-fold) overexpression of SirT1 attenuated age-dependent increases in oxidative stress. By contrast, high level (12.5-fold) overexpression of SirT1 resulted in increased levels of apoptosis and hypertrophy and decreased cardiac function. These observations indicated that there may be a negative regulator to resist the effects of SirT1. The elucidation of whether or not high levels of SirT1 result in destruction of the equilibrium mechanism requires further investigation.

In conclusion, SirT1 exerted negative effects on the regulation of STAT3 expression during oxidative stress. However, SirT1 and STAT3 each had protective roles against oxidative stress in RPEs. The results suggested that there may be an equilibrium mechanism between SirT1 and STAT3 against oxidative stress. This suggested that SIRT1 and STAT3 may be promising drugs for the treatment or prevention of AMD. However, there is equilibrium mechanism which must be considered when combined application of SIRT1 and STAT3 were performed.

\section{Acknowledgements}

The authors would like to thank Professor J Li (Beijing Institute of Microbiology and Epidemiology, Beijing, China) for valuable suggestions and advice. The present study was supported by the Renmin Hospital of Wuhan University (Wuchang, China) and Inner Mongolia People's Hospital (Hohhot, China).

\section{References}

1. Beatty S, Koh H, Phil M, Henson D and Boulton M: The role of oxidative stress in the pathogenesis of age-related macular degeneration. Surv Ophthalmol 45: 115-134, 2000.

2. Congdon NG, Friedman DS and Lietman T: Important causes of visual impairment in the world today. JAMA 290: 2057-2060, 2003.

3. Khan JC, Thurlby DA, Shahid H, et al: Smoking and age related macular degeneration: the number of pack years of cigarette smoking is a major determinant of risk for both geographic atrophy and choroidal neovascularisation. Br J Ophthalmol 90: 75-80, 2006.

4. Smith CJ and Hansch C: The relative toxicity of compounds in mainstream cigarette smoke condensate. Food Chem Toxicol 38 637-646, 2000.

5. Age-Related Eye Disease Study Research Group: A randomized, placebo-controlled, clinical trial of high-dose supplementation with vitamins $\mathrm{C}$ and $\mathrm{E}$ and beta carotene for age-related cataract and vision loss: AREDS report no. 9. Arch Ophthalmol 119: 1439-1452, 2001.

6. Crabb JW, Miyagi M, Gu X, et al: Drusen proteome analysis: an approach to the etiology of age-related macular degeneration. Proc Natl Acad Sci USA 99: 14682-14687, 2002.

7. Jarrett SG and Boulton ME: Consequences of oxidative stress in age-related macular degeneration. Mol Aspects Med 33: 399-417, 2012.

8. Plafker SM, O'Mealey GB and Szweda LI: Mechanisms for countering oxidative stress and damage in retinal pigment epithelium. Int Rev Cell Mol Biol 298: 135-177, 2012.
9. Wang ZY, Shen LJ, Tu L, et al: Erythropoietin protects retinal pigment epithelial cells from oxidative damage. Free Radic Biol Med 46: 1032-1041, 2009.

10. Cai J, Nelson KC, Wu M, Sternberg P Jr and Jones DP: Oxidative damage and protection of the RPE. Prog Retin Eye Res 19: 205-221, 2000

11. Li Z, Dong X, Liu H, et al: Astaxanthin protects ARPE-19 cells from oxidative stress via upregulation of Nrf2-regulated phase II enzymes through activation of PI3K/Akt. Mol Vis 19: 1656-1666, 2013.

12. Kook D, Wolf AH, Yu AL, et al: The protective effect of quercetin against oxidative stress in the human RPE in vitro. Invest Ophthalmol Vis Sci 49: 1712-1720, 2008.

13. Horio Y, Hayashi T, Kuno A and Kunimoto R: Cellular and molecular effects of sirtuins in health and disease. Clin Sci (Lond) 121: 191-203, 2011.

14. Raynes R, Brunquell J and Westerheide SD: Stress inducibility of SIRT1 and its role in cytoprotection and cancer. Genes Cancer 4: 172-182, 2013.

15. Alcendor RR, Gao S, Zhai P, et al: Sirt1 regulates aging and resistance to oxidative stress in the heart. Circ Res 100: 1512-1521, 2007.

16. Kawai Y, Garduno L, Theodore M, Yang J and Arinze IJ: Acetylation-deacetylation of the transcription factor Nrf2 (nuclear factor erythroid 2-related factor 2) regulates its transcriptional activity and nucleocytoplasmic localization. J Biol Chem 286: 7629-7640, 2011.

17. Levy DE and Lee CK: What does Stat3 do? J Clin Invest 109: $1143-1148,2002$.

18. Bourgeais J, Gouilleux-Gruart V and Gouilleux F: Oxidative metabolism in cancer: A STAT affair? JAKSTAT 2: e25764, 2013.

19. Zouein FA, Kurdi M and Booz GW: Dancing rhinos in stilettos: The amazing saga of the genomic and nongenomic actions of STAT3 in the heart. JAKSTAT 2: e24352, 2013.

20. Nie Y, Erion DM, Yuan Z, et al: STAT3 inhibition of gluconeogenesis is downregulated by SirT1. Nat Cell Biol 11: 492-500, 2009.

21. Bernier M, Paul RK, Martin-Montalvo A, et al: Negative regulation of STAT3 protein-mediated cellular respiration by SIRT1 protein. J Biol Chem 286: 19270-19279, 2011.

22. Polidoro L, Properzi G, Marampon F, et al: Vitamin D protects human endothelial cells from $\mathrm{H}_{2} \mathrm{O}_{2}$ oxidant injury through the Mek/Erk-Sirt1 axis activation. J Cardiovasc Transl Res 6: 221-231, 2013.

23. Li Y, Liu X, Zhou T, et al: Inhibition of APE1/Ref-1 redox activity rescues human retinal pigment epithelial cells from oxidative stress and reduces choroidal neovascularization. Redox Biol 2: 485-494, 2014.

24. Hori YS, Kuno A, Hosoda R and Horio Y: Regulation of FOXOs and $\mathrm{p} 53$ by SIRT1 modulators under oxidative stress. PLoS One 8: e73875, 2013

25. Kozlowski MR: RPE cell senescence: a key contributor to age-related macular degeneration. Med Hypotheses 78: 505-510, 2012.

26. Hanus J, Zhang H, Wang Z, Liu Q, Zhou Q and Wang S: Induction of necrotic cell death by oxidative stress in retinal pigment epithelial cells. Cell Death Dis 4: e965, 2013.

27. Yamada Y, Tian J, Yang Y, et al: Oxidized low density lipoproteins induce a pathologic response by retinal pigmented epithelial cells. J Neurochem 105: 1187-1197, 2008.

28. Patel AK and Hackam AS: Toll-like receptor 3 (TLR3) protects retinal pigmented epithelium (RPE) cells from oxidative stress through a STAT3-dependent mechanism. Mol Immunol 54: 122-131, 2013.

29. Geiger RC, Waters CM, Kamp DW and Glucksberg MR: KGF prevents oxygen-mediated damage in ARPE-19 cells. Invest Ophthalmol Vis Sci 46: 3435-3442, 2005.

30. Tanno M, Kuno A, Yano T, et al: Induction of manganese superoxide dismutase by nuclear translocation and activation of SIRT1 promotes cell survival in chronic heart failure. J Biol Chem 285: 8375-8382, 2010.

31. Kops GJ, Dansen TB, Polderman PE, et al: Forkhead transcription factor FOXO3a protects quiescent cells from oxidative stress. Nature 419: 316-321, 2002.

32. Vinciguerra M, Santini MP, Martinez C, et al: $\mathrm{mIGF-1/JNK1/SirT1}$ signaling confers protection against oxidative stress in the heart. Aging Cell 11: 139-149, 2012.

33. Caito S, Rajendrasozhan S, Cook S, et al: SIRT1 is a redox-sensitive deacetylase that is post-translationally modified by oxidants and carbonyl stress. FASEB J 24: 3145-3159, 2010. 
34. Arunachalam G, Yao H, Sundar IK, Caito S and Rahman I: SIRT1 regulates oxidant- and cigarette smoke-induced eNOS acetylation in endothelial cells: Role of resveratrol. Biochem Biophys Res Commun 393: 66-72, 2010

35. Yang SR, Wright J, Bauter M, Seweryniak K, Kode A and Rahman I: Sirtuin regulates cigarette smoke-induced proinflammatory mediator release via RelA/p65 NF-kappaB in macrophages in vitro and in rat lungs in vivo: implications for chronic inflammation and aging. Am J Physiol Lung Cell Mol Physiol 292: L567-L576, 2007.

36. Fasler-Kan E, Wunderlich K, Hildebrand P, Flammer J and Meyer P: Activated STAT3 in choroidal neovascular membranes of patients with age-related macular degeneration. Ophthalmologica 219: 214-221, 2005.

37. Zhang C, Li H, Liu MG, et al: STAT3 activation protects retinal ganglion cell layer neurons in response to stress. Exp Eye Res 86: 991-997, 2008.

38. Mimura T, Kaji Y, Noma H, Funatsu H and Okamoto S: The role of SIRT1 in ocular aging. Exp Eye Res 116: 17-26, 2013.
39. Cao L, Liu C, Wang F and Wang H: SIRT1 negatively regulates amyloid-beta-induced inflammation via the NF- $\mathrm{BB}$ pathway. Braz J Med Biol Res 46: 659-669, 2013.

40. Chou WW, Chen KC, Wang YS, Wang JY, Liang CL and Juo SH: The role of SIRT1/AKT/ERK pathway in ultraviolet $\mathrm{B}$ induced damage on human retinal pigment epithelial cells. Toxicol In Vitro 27: 1728-1736, 2013.

41. Scuto A, Kirschbaum M, Buettner R, et al: SIRT1 activation enhances HDAC inhibition-mediated upregulation of GADD45G by repressing the binding of NF- $\mathrm{KB} / \mathrm{STAT} 3$ complex to its promoter in malignant lymphoid cells. Cell Death Dis 4: e635, 2013.

42. Borden EC, Sen GC, Uze G, et al: Interferons at age 50: past, current and future impact on biomedicine. Nat Rev Drug Discov 6: 975-990, 2007.

43. Yuan ZL, Guan YJ, Chatterjee D and Chin YE: Stat3 dimerization regulated by reversible acetylation of a single lysine residue. Science 307: 269-273, 2005. 